\title{
Thermovoltaic Effect in a Multilayer Junction Structure with an Oxide Insulation Barrier
}

\section{Marta Turkiewicz, Eugeniusz Prociów, Andrzej Dziedzic}

Faculty of Microsystem Electronics and Photonics, Wrocław University of Science and Technology, Wybrzeże Wyspiańskiego 27, 50-370 Wrocław, Poland, marta.turkiewicz@pwr.edu.pl and andrzej.dziedzic@pwr.edu.pl

\begin{abstract}
The thermovoltaic effect describes the formation of electromotive force (EMF) in the absence of an external temperature gradient (in contrast to the conventional Seebeck phenomenon, which requires the creation of a temperature gradient between two semiconductor or metal connectors). In the presented research, the structure of a microgenerator using a thermovoltaic effect was proposed, in which the transport of hot electrons through an oxide insulating barrier lead to the accumulation of charge carriers and the formation of inter-layer potential. The microgenerator structures were fabricated in a multi-stage magnetron sputtering process. Planar structures were fabricated, consisting of thin layers of heavily doped degenerate semiconductor (SiC:Zr:B, Ge:Au, $\left.\mathrm{Ge}: \mathrm{Ta}: \mathrm{V}, \mathrm{TiO}_{2}: \mathrm{Nb}\right)$ with a silicon dioxide $\left(\mathrm{SiO}_{2}\right)$ insulation barrier and a second siliconmetal based electrode ( $\mathrm{Ta}, \mathrm{Mo}, \mathrm{NiCr}$ ). The thermovoltaic effect was observed in all of the structures, and the value of the generated electromotive force (thermovoltaic voltage) and the value of effective power was determined. The presented preliminary results show that the concept of constructing of asymmetric multi-layer junction structures can be used to fabricate microgenerators using a thermovoltaic effect.
\end{abstract}

Keywords: degenerated semiconductor; insulating barrier; thermovoltaic effect

\section{Introduction}

In recent years, energy harvesting has become an important issue, both on a micro- and macro scale. The aim of such studies is to transform the energy available in the ambient into useable electrical energy based on various physical phenomena. For example, the thermoelectric phenomena or the photovoltaic effect are well known and used on the industrial scale. Much less information concerns the thermovoltaic effect presented in this article. It permits for the direct conversion of thermal energy into electricity, but unlike the conventional Seebeck effect, the electromotive force is generated here without the presence of the temperature gradient. 
In general, in a thermovoltaic phenomenon, voltage generation occurs without the need to produce an external temperature gradient in the structure, only during uniform heating of the structure. For the first time, this effect was observed in a polycrystalline sample of samarium sulphide $(\mathrm{SmS})[1,2]$. In this structure, the generation of electromotive force resulted directly from the artificial creation of a gradient of excess samarium ions in a parallelepipedal SmS polycrystalline sample. Subsequently, thermovoltaic effect was discovered in other materials SmS-based heterostructures [3], ZnO-based compositions [4, 5] as well as $\mathrm{Si}$ - and Ge-based structures [6].

In the classic linear transport regime there is a fundamental difference between a large value Seebeck coefficient and high electrical conductivity for electrical materials conductive and many multilayer structures due to the interaction between electron density of states (DOS) and the speed of electron groups, and also due to shape of the energy distribution curve. It is known that lowdimensional structures change the density of states. For high barrier height and high doping concentration, semiconductor heat energy converters would be able to reduce this effect, thus achieving a high thermoelectric power efficiency-factor.

To obtain this effect there cannot be saved electron transverse momentum perpendicular to heterostructural barriers. This can be achieved using non-flat structures or embedded nanostructures. A comparison of thermoelectric devices and thermoelectric energy converters shows a difference in the average energy of emitted hot carriers due to the difference between the density of electron and photon states in the tested material. The use of both electrons and photons from the hot reservoir part or state density engineering can provide additional conditioning for achieving higher performance in energy conversion devices and further approaching the limit obtained by entropy generation.

The introduction of potential barriers in highly doped semiconductors or metals can increase the asymmetry between cold and hot electron transport, which could eliminate conventional optimization between electrical conductivity and the Seebeck coefficient. It is considered that not all hot electrons with energy greater than barrier height are transported above the barrier. Because of the electron states in space momentum at planar barriers, only electrons with kinetic energy higher than the threshold value can be emitted perpendicular to the barrier (Fig. 1). In the case of a planar barrier, hot electrons moving under a large one angle to the interface are completely reflected internally.

The conservation of the transverse momentum results from the symmetry of the system from the invariability of translation in a direction perpendicular to the barrier layers. By using non-flat barriers or embedded nanostructures, this symmetry can be broken.

A further beneficial technological activity in the construction of the produced layer would be breaking the symmetry without a significant reduction of the electron mean free path and mobility of electrons in the structure. 


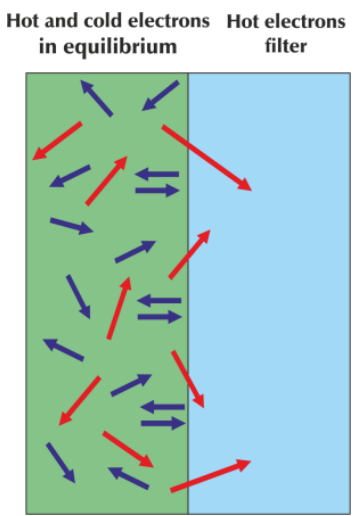

Figure 1

Graphic diagram of hot and cold electrons in equilibrium

For this purpose, high crystallinity is required close to the interface with low defect density. This is achieved by using, for example, embedded nanoparticles. If a transverse momentum block is formed, not only is the significant reduction in the number of electrons emitted, but also energy filtering is not rapid even with thick barriers. There is also a major compromise between the electron density of states and the speed of electron groups in crystalline solids. This is manifested in the fact that solids with a high effective mass of electrons have a high density of states, but at the same time have lower mobility. The electron velocity group is related to the derivative of the dispersion dependence (electron energy concerning its momentum), while the state density is related to the inverse of the energy band curvature. The shape of the density of states dominates in thermoelectric and thermonic devices and materials with heavy effective electron masses and many valleys high potentials at high temperatures.

In the presented research, the structure of a microgenerator using a thermovoltaic effect was proposed, in which the transport of hot electrons through an oxide insulating barrier lead to the accumulation of charge carriers and the formation of inter-layer potential. The design of the generators based on the thermovoltaic effect allowed for the generation of electric voltage without the need to create a temperature gradient in the structure. The first work on thin-film generators showed so promising results that it may be concluded that it is possible to build miniature energy generators using an effect other than the classic approach based on the Seebeck effect. Not requiring a temperature difference in the structure significantly simplifies the design of generator systems. The issues related to thermodynamics can thus be narrowed down to homogeneous annealing of the entire structure using a simple heating element and removing heat from the structure. 
The presented research is the result of an experiment on real structures fabricated in multi-stage technological processes. The generator design was proposed based on own previous studies of thin-film thermoelectric elements and the general concept of microgenerators' fabrication.

The paper is organized as follows. The analysis of the literature in the field was presented in the Introduction. The materials, technology and general construction of investigated multilayered thermovoltaic microgenerators are described in Chapter 2. The setup for measurement of electromotive force for structures homogeneously heated from ambient temperature up to about $573 \mathrm{~K}$ is given in Chapter 3. The electrical properties, expressed by experimental characteristics and fitting curves of electromotive force and effective power vs isothermal temperature of multilayer thermovoltaic microgenerator, are shown in Chapter 4. The conclusions are pointed out in Chapter 5 .

\section{Multilayered Thermovoltaic Generator}

Based on the above considerations, a microgenerator was fabricated using a ballistic passage of hot electrons through the insulation barrier. This transition causes the accumulation of charge and the emergence of inter-layer potential. Several planar structures were made, fabricated of a thin-film multilayer of a degenerated semiconductor with an oxide nanobarrier and a second layer based on selected metal silicide. Highly doped semiconductors such as SiC:Zr:B, Ge:Au, $\mathrm{Ge}: \mathrm{Ta}: \mathrm{V}$, or $\mathrm{TiO}_{2}: \mathrm{Nb}$ were used as an active material. One should add that previously SiC:Zr:B films were tested as NTC thermistors [7] whereas Ge:Au, Ge:Ta: $\mathrm{V}$ and $\mathrm{TiO}_{2}: \mathrm{Nb}$ - as thermoelectric materials $[8,9]$. The second electrode was made as to metal silicide - one of $\mathrm{Ta}$, Mo and $\mathrm{NiCr}$. The scheme of construction of a multilayer double junction thermovoltaic microgenerator is shown in Fig. 2.

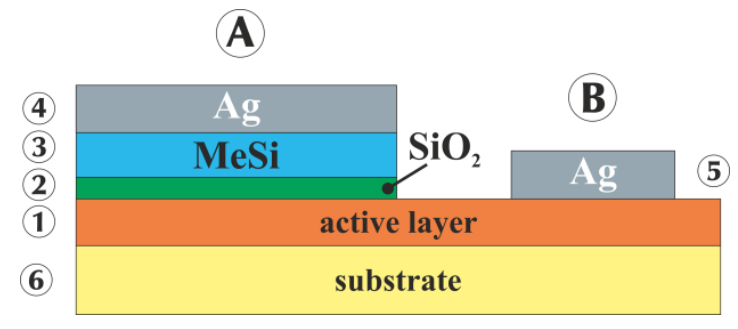

Figure 2

Scheme of construction of a multilayer double junction thermovoltaic microgenerator (thermocouple

A: 1 - thin film semiconductive active layer, 2 - oxide barrier, 3 - buffer layer MeSi, 4 - Ag contact layer; thermocouple B: 1 - thin film semiconductive active layer, 5 - Ag contact layer) 
All thin films used for the construction of the microgenerators' active area were produced in energetically sublime magnetron sputtering processes using the Pfeiffer Classic 570 vacuum system with WMK-100 magnetrons. Thin layers were deposited onto high temperature $(573 \mathrm{~K})$ Corning 7059 glass substrates. Films were fabricated with the use of alloy or mosaic targets or in a co-sputtering process with the use of two different targets onto two magnetrons. Besides, magnetron sputtering processes were carried out under conditions of minimal pressure, conditioned by the specificity of the plasma process. Thin layers were applied in an argon atmosphere (except for layers $\mathrm{TiO}_{2}: \mathrm{Nb}$, applied in an argonoxygen atmosphere), while maintaining a constant operating pressure $p \leq 10^{-3} \mathrm{Tr}$, which allowed to obtain anode-cathode discharge voltage with a voltage above $680 \mathrm{~V}$. Such conditions of the plasma process determined the appropriate energy conditions for structures punched from the target surface and forming a thin semiconductor layer.

The active layers were characterized by a polycrystalline structure with a high degree of microstructure organization and the thickness in a range of $0.4-4 \mu \mathrm{m}$. Besides, they showed the dominant type of electrical conductivity (hole or electron) depending on the magnetron sputtering conditions and dopant used. What's more, it was confirmed that by choosing the right concentration of dopant material and the post-process treatment, it is possible to optimize the electrical properties of thin active layers and their subsequent power efficiency. After deposition, semiconductor active layers were annealed at $573 \mathrm{~K}$ in air.

To form the microgenerator structure, on the surface of the active semiconductive layer, a silicide $\left(\mathrm{TaSi}_{2}, \mathrm{MoSi}_{2}, \mathrm{NiCrSi}\right.$ ) layer was also applied in the magnetron sputtering process. Silicon-metal targets were used to make the silicide films, which were characterized by thicknesses in the range of 4-6 $\mu \mathrm{m}$.

During the deposition of silicide, a nanometric layer of silicon dioxide occurred spontaneously on the border between the active layer of the semiconductor and the silicide layer. It is worth to briefly explain the mechanism of this insulation layer formation. It is known that a silicon dioxide layer is formed on the surface of metal silicides in an oxygen atmosphere $[10,11]$. During the thermal post-process treatment of the active layer, a semiconductor oxide layer is formed on its surface [12]. In this way, oxygen appears in the area between semiconductor and silicide, which is then supplied to produce silicon dioxide (Fig. 3).

Silver electrodes were applied to the semiconductor and silicide layers to make electrical contacts. The electrodes were prepared in the thermal evaporation process. 
1) annealed active layer

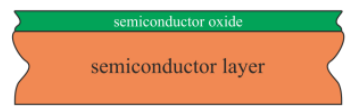

2) $\mathrm{SiO}_{2}$ layer formation

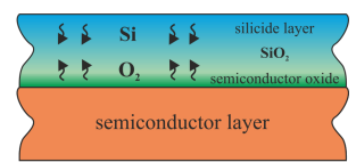

Figure 3
3) $\mathrm{MeSi} / \mathrm{SiO}_{2} /$ active layer structure

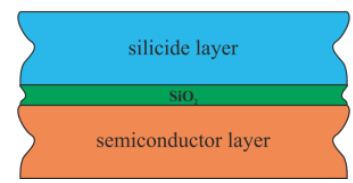

Mechanism of insulating oxide barrier formation

In the proposed construction of a multilayer two-junction microgenerator the junctions, in contrast to the classic thermocouple, were placed at a mutual distance of less than $1 \mathrm{~mm}$. The total area of the microgenerator did not exceed $0.25 \mathrm{~cm}^{2}$, including the area of thermocouples, which was $2 \mathrm{~mm}^{2}$ and $1 \mathrm{~mm}^{2}$ for thermocouple A and B, respectively.

\section{Measurement Setup}

Test measurements of the fabricated microgenerators were carried out under the conditions of isothermal heating of structures, and to compare thermoelectric properties - also with gradient heating of the structure. To maintain isothermal conditions, the surface of the heated plate was around $20 \mathrm{~cm}^{2}$ i.e. much more than the total surface of the microgenerator. Symmetrical probes and electrical connections (Ag or $\mathrm{CuSnP}$ ) were used to the peripheral measuring system. Measurements of electrical properties were carried out in open circuit conditions loading microgenerators with the input resistance of the Fluke 8846A multimeter voltmeter, and the current-voltage characteristics at the generator load with variable resistance. Current efficiency measurements were carried out at a constant generator load with a resistance value of $R=1 \Omega$. All of the measurements were made without the use of external power sources, treating the microgenerator as a current-voltage source of the measuring system. The microgenerator structure is shown in Fig. 4. 


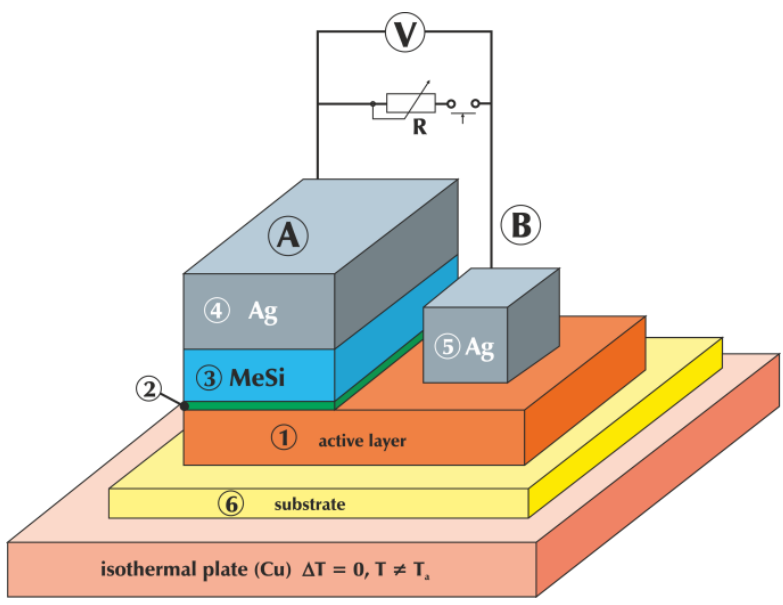

Figure 4

Scheme of construction of a multilayer double junction thermovoltaic microgenerator (in a test setup to measure thermovoltaic properties) (thermocouple A: 1 - thin film semiconductive active layer, 2 oxide barrier, 3 - buffer layer MeSi, 4 - Ag contact layer: thermocouple B: 1 - thin film semiconductive active layer, 5 - Ag contact layer).

\section{Electrical and Thermoelectric Properties}

To compare the tested structures with each other, the effective power $P_{e}$ was defined, i.e. the product of the short-circuit current $I$ and the electromotive force $E M F$ of the open circuit concerning the active surface $A$ occupied by the thermocouple (Equation 1).

$$
P_{e}=\frac{E M F \cdot I}{A}
$$

For all of the tested microgenerators the electromotive force (or thermovoltaic voltage) was determined in the temperature range from $323 \mathrm{~K}$ to $553 \mathrm{~K}$. The effective power $P_{\mathrm{e}}$ was determined for all generators, except for the structure with the $\mathrm{TiO}_{2}: \mathrm{Nb}$ active layer, for which, on the other hand, the $E M F_{\mathrm{TE}}$ value generated as a result of the classic Seebeck effect (with the external temperature gradient) was compared with the value of the thermovoltaic voltage $E M F_{\mathrm{TV}}$.

It was assumed that linear function describes the dependence of electromotive force vs temperature of microgenerator (formally similar as for Seebeck effect, but one should remember that in thermovoltaic effect we have a temperature of the whole structure whereas for Seebeck effect we have a temperature gradient between hot and cold junctions) and exponential function describe effective power 
vs temperature. Due to the lack of a commonly accepted physical model of the thermovoltaic phenomenon, it is impossible to apply an advanced model (shown eg. in [13-15]) for the approximation of experimental characteristics.

\subsection{Ge:Au Active Layer}

$\mathrm{Ge}: \mathrm{Au}$ active thin films, with a thickness of approximately $1.63 \mu \mathrm{m}$, were fabricated from the germanium-gold alloy target with $5 \mathrm{wt} \%$ of Au. The resistivity of Ge:Au films were measured by the four-probe method and was around 0.059 $\Omega \mathrm{cm}$. Figure 5 presents the relationship between the electromotive force $E M F_{\mathrm{TV}}$ generated in an $\mathrm{Ag} / \mathrm{Ge}: \mathrm{Au} / \mathrm{SiO} 2 / \mathrm{NiCrSi} / \mathrm{Ag}$ structure after uniform heating it to the temperature $T$. The generated force increased with increasing structure's temperature, reaching the highest value $E M F_{\mathrm{Ge}: \mathrm{Au}}=8.22 \mathrm{mV}$ for $\mathrm{T}=550 \mathrm{~K}$.

The dependence of effective power $P_{\mathrm{e}}$ on the temperature for microgenerator with Ge:Au active film was determined as well (Fig. 6). The $P_{\mathrm{e}}$ value increased with increasing structure temperature was with the maximum value of $61.53 \mu \mathrm{W} / \mathrm{cm}^{2}$ at $500 \mathrm{~K}$.

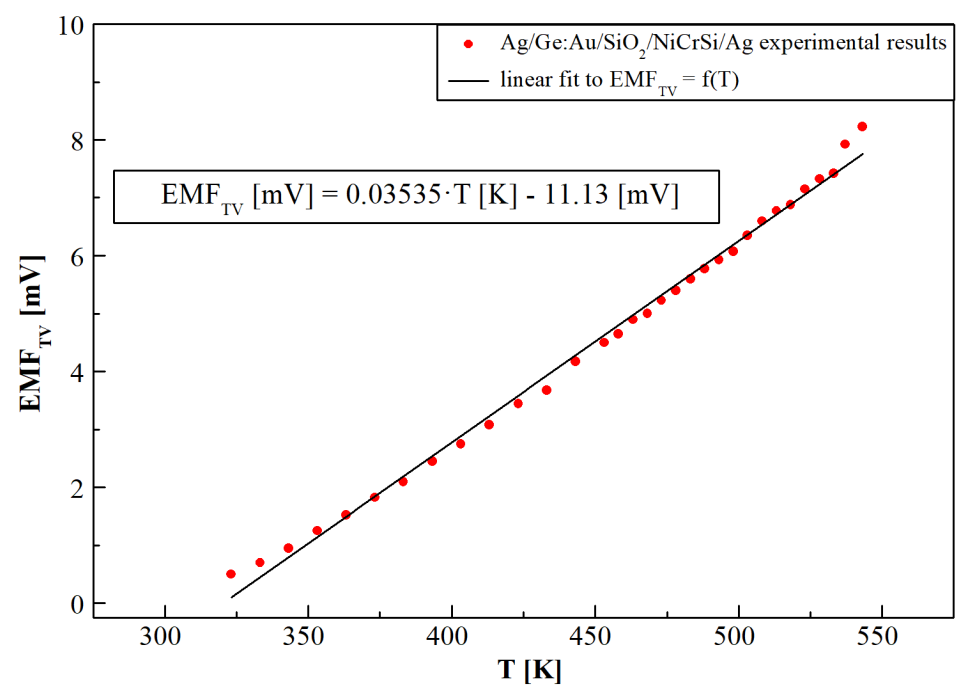

Figure 5

Dependence of electromotive force $E M F_{\mathrm{TV}}$ (thermovoltaic voltage) on the temperature of the microgenerator with $\mathrm{Ag} / \mathrm{Ge}: \mathrm{Au} / \mathrm{SiO}_{2} / \mathrm{NiCrSi} / \mathrm{Ag}$ structure 


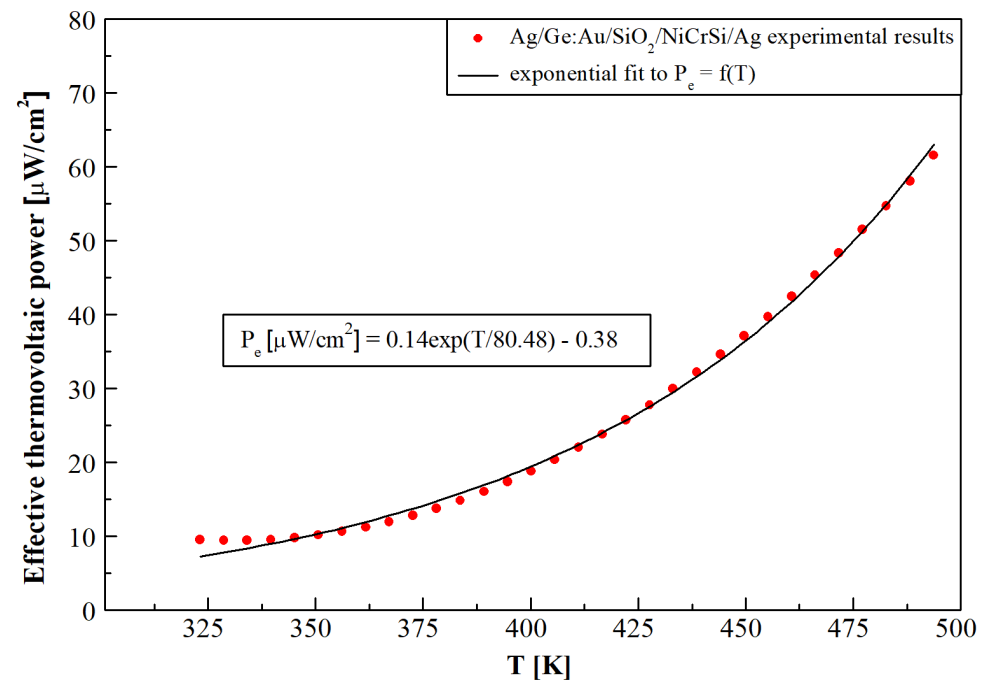

Figure 6

Dependence of effective power $P_{\mathrm{e}}$ on the temperature of the microgenerator with $\mathrm{Ag} / \mathrm{Ge}: \mathrm{Au} / \mathrm{SiO}_{2} / \mathrm{NiCrSi} / \mathrm{Ag}$ structure

\subsection{Ge:Ta:V Active Layer}

The Ge:Ta: $\mathrm{V}$ target was made from the germanium-tantalum alloy target. Composition with vanadium was achieved by partially covering the Ge:Ta target surface with triangular-shaped tantalum pieces. The composition of Ge:Ta:V films, measured by EDS-XRD microscopy, was 88 at.\% Ge, 9.5 at.\% V, and 2.5 at.\% Ta. The thickness of Ge:Ta:V films was about $2.1 \mu \mathrm{m}$ and the resistivity measured by the four-probe method and was around $6.5 \cdot 10^{-3} \Omega \mathrm{cm}$. Figure 7 presents the relationship between the electromotive force $E M F_{\mathrm{TV}}$ generated in an $\mathrm{Ag} / \mathrm{Ge}: \mathrm{Ta}: \mathrm{V} / \mathrm{SiO}_{2} / \mathrm{NiCrSi} / \mathrm{Ag}$ structure after uniform heating it to the temperature $T$. The generated force increased with increasing structure's temperature, reaching the highest value $E M F_{\mathrm{Ge}: \mathrm{Ta}: \mathrm{V}}=3.50 \mathrm{mV}$ for $\mathrm{T}=550 \mathrm{~K}$.

The dependence of effective power $P_{\mathrm{e}}$ on the temperature of a microgenerator with Ge:Ta:V active film was determined as well (Fig. 8). The $P_{\mathrm{e}}$ value was also higher for the higher structure temperature, with the maximum value of 170 $\mu \mathrm{W} / \mathrm{cm}^{2}$ at $550 \mathrm{~K}$. 


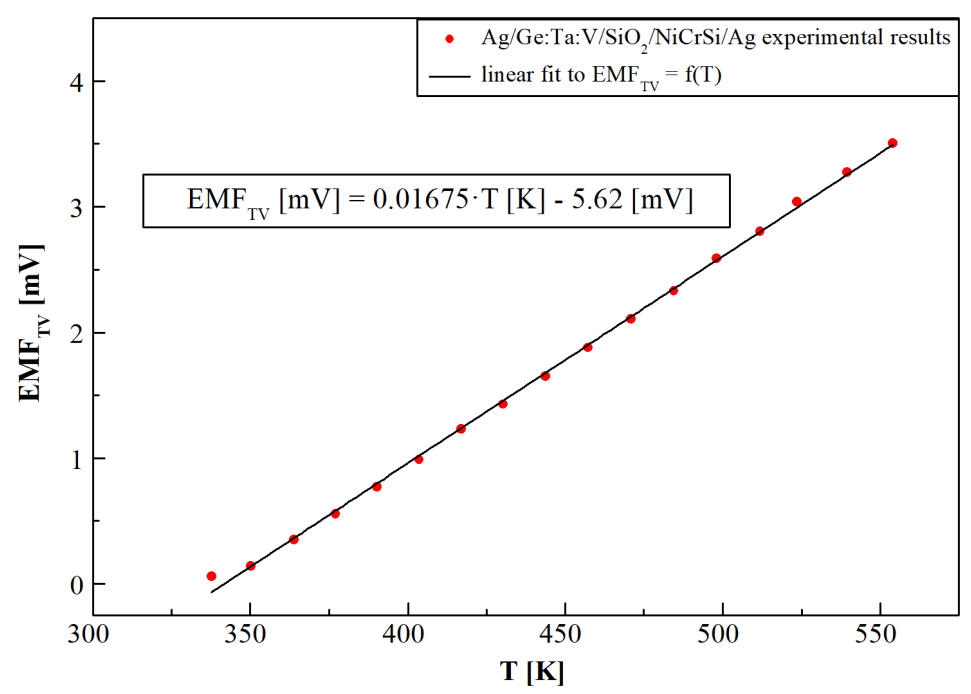

Figure 7

Dependence of electromotive force $E M F_{\mathrm{TV}}$ (thermovoltaic voltage) on the temperature of the microgenerator with $\mathrm{Ag} / \mathrm{Ge}: \mathrm{Ta}: \mathrm{V} / \mathrm{SiO}_{2} / \mathrm{NiCrSi} / \mathrm{Ag}$ structure

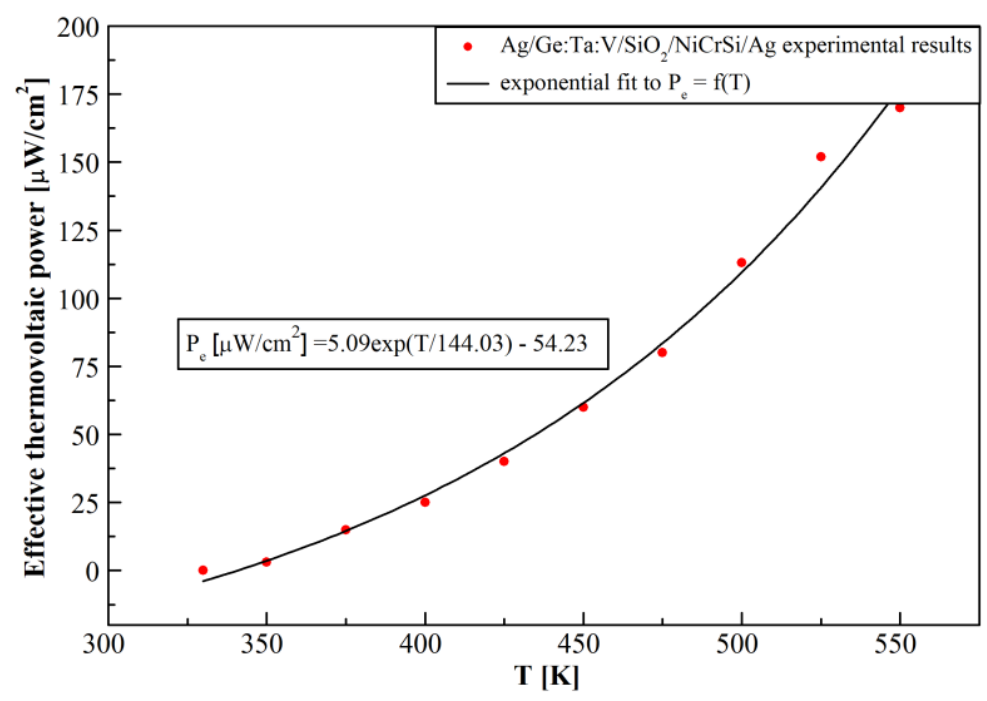

Figure 8

Dependence of effective power $P_{\mathrm{e}}$ on the temperature of the microgenerator with $\mathrm{Ag} / \mathrm{Ge}: \mathrm{Ta}: \mathrm{V} / \mathrm{SiO}_{2} / \mathrm{NiCrSi} / \mathrm{Ag}$ structure 


\subsection{SiC:Zr:B Active Layer}

$\mathrm{SiC}: \mathrm{Zr}: \mathrm{B}$ active thin films were fabricated in the co-sputtering process. After the process $\mathrm{SiCZr}_{0.07}: \mathrm{B}_{0.12}$ layer was received. The thickness of films was approximately $4.05 \mu \mathrm{m}$ and they were characterized by resistivity $\rho_{\mathrm{SiC}: \mathrm{Zr}: \mathrm{B}}=0.04$ $\Omega \mathrm{cm}$ (measured by four-probe method). Figure 9 presents the relationship between the electromotive force $E M F_{\mathrm{TV}}$ generated for $\mathrm{Ag} / \mathrm{SiC}: \mathrm{Zr}: \mathrm{B} / \mathrm{SiO}_{2} / \mathrm{NiCrSi} / \mathrm{Ag}$ structure after uniform heating it to the temperature $T$. The generated force increased with increasing structure's temperature, reaching the highest value $E M F_{\mathrm{SiC}: \mathrm{Zr}: \mathrm{B}}=4.18 \mathrm{mV}$ for $\mathrm{T}=550 \mathrm{~K}$.

The dependence of effective power $P_{e}$ on the temperature of a microgenerator with SiC:Zr:B active film was determined as well (Fig. 10). The higher the $P_{e}$ value, the higher the structure temperature was, with the maximum value of 380 $\mu \mathrm{W} / \mathrm{cm}^{2}$ at $550 \mathrm{~K}$, and it was the highest among all tested structures.

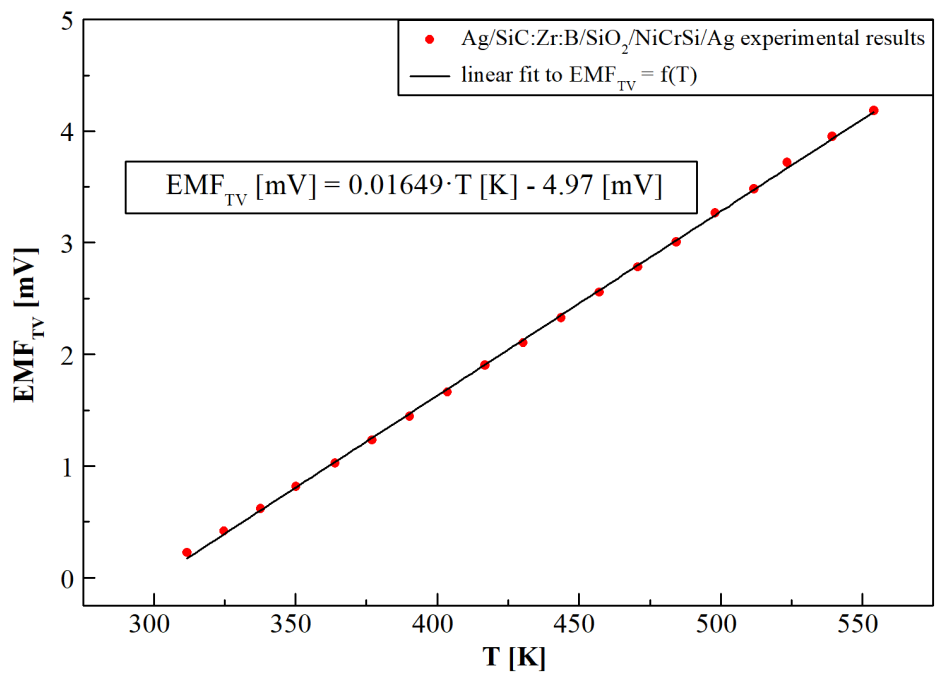

Figure 9

Dependence of electromotive force $E M F_{\mathrm{TV}}$ (thermovoltaic voltage) on the temperature of the microgenerator with $\mathrm{Ag} / \mathrm{SiC}: \mathrm{Zr}: \mathrm{B} / \mathrm{SiO}_{2} / \mathrm{NiCrSi} / \mathrm{Ag}$ structure 


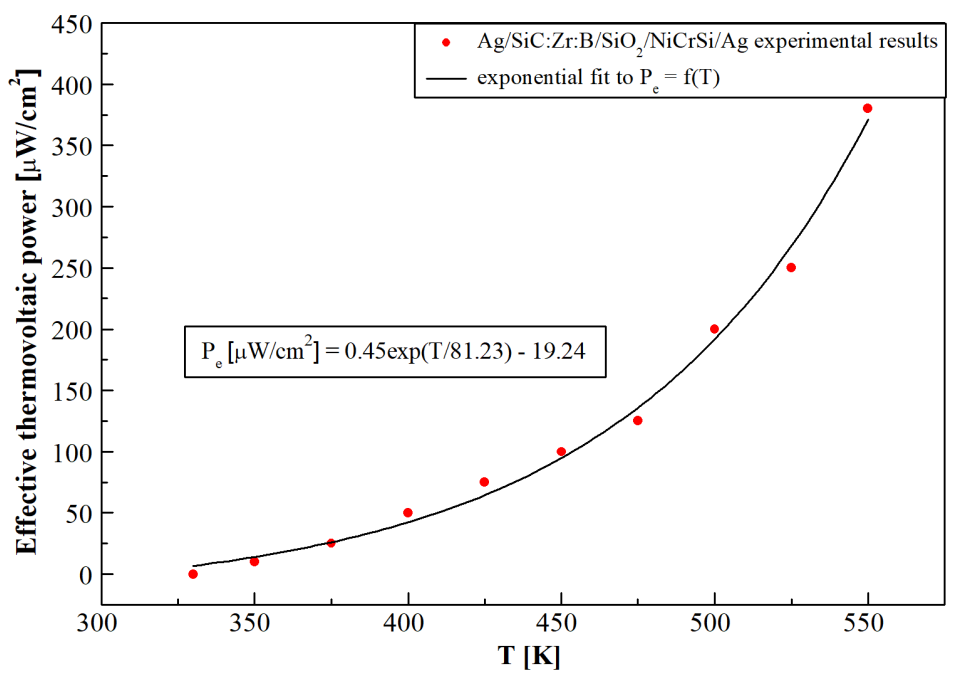

Figure 10

Dependence of effective power $P_{\mathrm{e}}$ on the temperature of the microgenerator with $\mathrm{Ag} / \mathrm{SiC}: \mathrm{Zr}: \mathrm{B} / \mathrm{SiO}_{2} / \mathrm{NiCrSi} / \mathrm{Ag}$ structure

\section{4 $\mathrm{TiO}_{2}$ : $\mathrm{Nb}$ Active Layer}

$\mathrm{TiO}_{2}: \mathrm{Nb}$ active thin films were fabricated from the $\mathrm{TiO}_{2}: \mathrm{Nb}$ mosaic target $(5$ at. $\%$ $\mathrm{Nb}$ in $\mathrm{TiO}_{2}$ ). The thickness of active films were approximately $0.38 \mu \mathrm{m}$, and the resistivity of $\mathrm{TiO}_{2}: \mathrm{Nb}$ films was around $2.47 \cdot 10^{-3} \Omega \mathrm{cm}$ (measured by four-probe method).

For microgenerator with a $\mathrm{TiO}_{2}: \mathrm{Nb}$ active layer, it was examined whether there was a difference between the value of the electromotive force generated in the structure between the classic Seebeck effect and the thermovoltaic effect (Fig. 11). It has been shown that the generated electromotive force $E M F$ assumes similar values in both cases - both when measuring the structure in which the temperature gradient $\left(E M F_{\mathrm{TE}}\right)$ was created and when measuring the uniformly heated structure $\left(E M F_{\mathrm{TV}}\right)$.

One should notice that similarly designed Pt silicide/silicon multilayer structured device with 3 to $12 \mathrm{PtSi} / \mathrm{Si}$ heterojunctions (i.e. without oxide insulation layer) exhibited classic Seebeck effect [16] and was used for fabrication of 3D thermoelectric energy generator consisting of 127 pairs of such legs [17]. 


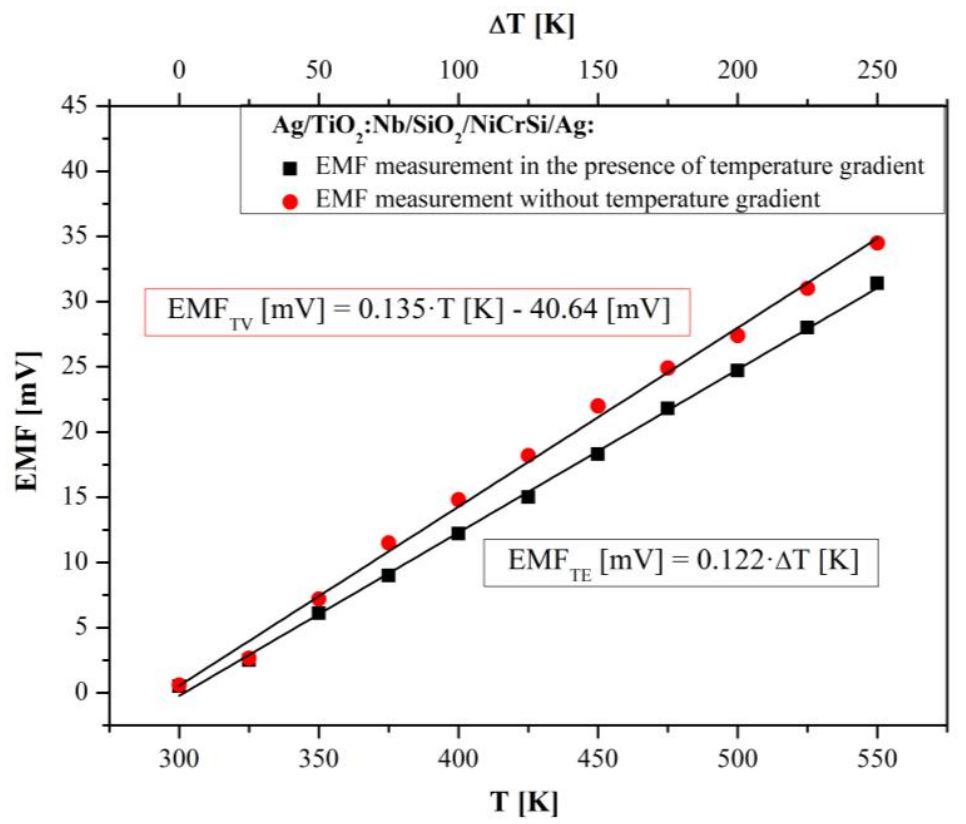

Figure 11

Dependence of electromotive force $E M F$ (thermovoltaic or thermoelectric voltage) on the temperature $T$ of the microgenerator with $\mathrm{Ag} / \mathrm{TiO}_{2}: \mathrm{Nb} / \mathrm{SiO}_{2} / \mathrm{NiCrSi} / \mathrm{Ag}$ (thermovoltaic effect) and on the temperature gradient $\Delta T$ created between thermocouple joints (conventional Seebeck effect)

\section{Results and Discussion}

Using technological processes based on high-energy and low-pressure magnetron sputtering, it is possible to obtain functional thin semiconductive films with ordered structure and desired electrical and thermoelectric properties.

The generation of thermovoltaic voltage was observed both for semiconductor layers with large $\left(\mathrm{TiO}_{2}: \mathrm{Nb}\right)$ and small $(\mathrm{Ge}: \mathrm{Au})$ bandgap. The $E M F_{\mathrm{TV}}$ value depended only on the type of conductivity obtained in the semiconductor layer. The highest value of effective power was obtained for the $\mathrm{Ag} / \mathrm{SiC}: \mathrm{Zr}: \mathrm{B} / \mathrm{SiO}_{2} / \mathrm{NiCrSi} / \mathrm{Ag}$ structures. It was several $\mu \mathrm{W} / \mathrm{cm}^{2}$ at ambient temperature and increased exponentially to around $200 \mu \mathrm{W} / \mathrm{cm}^{2}$ at $500 \mathrm{~K}$. About half the smaller effective power values were obtained for microgenerators with a $\mathrm{Ge}: \mathrm{Ta}: \mathrm{V}$ active layer - from several $\mu \mathrm{W} / \mathrm{cm}^{2}$ at ambient temperature to 113 $\mu \mathrm{W} / \mathrm{cm}^{2}$ at $500 \mathrm{~K}$. Such measurements can lead to the conclusion that obtaining higher effective power values is possible for multi-component compounds (in this case ternary) of highly degenerated semiconductors. What's important, the high 
values of effective power were obtained both for the microgenerators with active layers with $n$-type (SiC:Zr:B) and $p$-type (Ge:Ta:V) electrical conductivity.

What's interesting, a similar $E M F$ value was measured for the structures, in which the temperature gradient was created (classic Seebeck effect) and the structures uniformly heated on an isothermal plate (thermovoltaic effect). However, for possible applications, operation without the need for the creation of an artificial temperature gradient in the structure should allow for increased thermovoltaic power, larger efficiency, and elimination of heating elements in the system. Such thermovoltaic microgenerators require only one heating element, which limits the number of elements in the system, reduces the number of heat sinks that remove heat from the generator, and significantly affects the simplicity of mounting microgenerators in the systems with which they are to cooperate. The use of the classic Seebeck effect makes it necessary to linearly lengthen the structure between thermal joints and optimize the thermal and energy properties of the structure that affect the power efficiency of the thermogenerator. On the other hand, thermovoltaic microgenerators are made in the form of thin-film multilayers, which affects the degree of complexity of the technological process leading to their implementation. As shown in the article, the desired electrical and thermoelectric properties can be obtained only for structures made in high-energy and low-pressure magnetron sputtering processes.

\section{Conclusions}

The designs of several microgenerators utilizing the thermovoltaic effect - the generation of electromotive force $E M F_{\mathrm{TV}}$ in the absence of a temperature gradient in the structure, and only under conditions of its homogeneous heating, have been described. The proposed microgenerators were fabricated in the form of thin-film multilayer structures, sputtered in the process of low-pressure and high-energy magnetron sputtering. The microgenerators differed in the active layer material $\mathrm{SiC}: \mathrm{Zr}: \mathrm{B}, \mathrm{Ge}: \mathrm{Au}, \mathrm{Ge}: \mathrm{Ta}: \mathrm{V}, \mathrm{TiO}_{2}: \mathrm{Nb}$ thin films were used. All microgenerators enabled the generation of electromotive force with values in the range from several to several dozen $\mathrm{mV}$ - changing with the temperature of the structure. The proposed concept and the constructed structures constitute a new contribution to the group of research focused on the generation of electricity in miniature structures. The use of the thermovoltaic effect is a new, another approach to building generators.

\section{Acknowledgment}

This work was supported by the statutory grant of Wrockaw University of Science and Technology.

\section{References}

[1] V. V. Kaminskii et al.: Thermovoltaic Effect in Polycrystalline Samarium Sulfide, Technical Physics Letters, Vol. 35, 2009, pp. 981-984 
[2] V. V. Kaminskii: Dynamics of the Thermovoltaic Effect in SmS, Technical Physics Letters, Vol. 39, 2013, pp. 673-675

[3] V. V. Kaminskii et al.: Thermovoltaic Effect in Samarium Sulfide-Based Heterostructures, Technical Physics, Vol. 56, 2011, pp. 893-895

[4] I. A. Pronin et al.: The Thermovoltaic Effect in Zinc Oxide Inhomogeneously Doped with Mixed-Valence Impurities, Technical Physics Letters, Vol. 41, 10, 2015, pp. 930-932

[5] I. A. Pronin et al.: Development of a Physical Model of Thermovoltaic Effects in the Thin Films of Zinc Oxide Doped with Transitions Metals, Coatings, Vol. 8, 2018, 433 (12 pp.)

[6] A. S. Saidov, A. Yu. Leyderman, A. B. Karshiev: The thermovoltaic effect in variband solid solution $\mathrm{Si}_{1-\mathrm{x}} \mathrm{Ge}_{\mathrm{x}}(0 \leq \mathrm{x} \leq 1)$, Technical Physics Letters, Vol. 42, 2016, pp. 725-728

[7] A. Dziedzic, E. L. Prociów: Some Remarks about Planar Thermistors, Proc. $25^{\text {th }}$ Int. Spring Seminar on Electronics Technology, Prague (Czech Republic), May 2002, pp. 114-117

[8] P. Markowski, E. Prociów, A. Dziedzic: Mixed Thick/Thin-Film Thermocouples for Thermoelectric Microgenerators and Laser Power Sensor, Optica Applicata, Vol. 39, 2009, pp. 681-690

[9] E. Prociów, A. Dziedzic: Influence of Thermal Treatment on Electrical Properties of Transparent $\mathrm{TiO}_{2}: \mathrm{Nb}$ Thin Films, Proc. $39^{\text {th }}$ Int. Spring Seminar on Electronics Technology, Pilsen (Czechia), May 2016, pp. 87-90

[10] L. N. Lie, W. A. Tiller, K. C. Saraswat: Thermal Oxidation of Silicides, Journal of Applied Physics, Vol. 56, 1984, pp. 2127-2132

[11] B. E. Deal, A. S. Grove: General Relationship for the Thermal Oxidation of Silicon, Journal of Applied Physics, Vol. 36, 1965, pp. 3770-3778

[12] J. Oh, J. C. Campbell: Thermal Desorption of Ge Native Oxides and Loss of Ge from the Surface, Materials Science in Semiconductor Processing, Vol. 13, 2010, pp. 185-188

[13] J. C. Spall: Multivariate Stochastic Approximation Using a Simultaneous Perturbation Gradient Approximation, IEEE Transaction on Automatic Control, Vol. 37, 1992, pp. 332-341

[14] T. Haidegger et al.: Cascade Control for Telerobotic Systems Serving Space Medicine, Proc. $18^{\text {th }}$ IFAC (The International Federation of Automatic Control) World Congress, Milano (Italy), 2011, pp. 3759-3764

[15] E.-L. Hedrea, R.-E. Precup, C.-A. Bojan-Dragos: Results on Tensor Product-based Model Transformation on Magnetic Levitation Systems, Acta Polytechnica Hungarica, Vol. 16, No. 9, 2019, pp. 93-111 
[16] W. Choi et al.: Thermoelectric Characteristics of Pt-Silicide/Silicon MultiLayer Structured p-Type Silicon, Energy, Vol. 82, 2015, pp. 180-183

[17] J.-P. Im et al.: Self-Powered Autonomous Wireless Sensor Node by Using Silicon-Based 3D Thermoelectric Energy Generator for Environmental Monitoring Application, Energies, Vol. 13, 2020, 674 (17 pp.) 